\title{
In Situ Micropillar Compression of Irradiated HT9
}

Ryan Schoell ${ }^{1}$, Ce Zheng ${ }^{1}$, Khalid Hattar ${ }^{2}$ and Djamel Kaoumi ${ }^{1}$

${ }^{1}$ North Carolina State University, Raleigh, North Carolina, United States, ${ }^{2}$ Sandai National Laboratories, Albuquerque, New Mexico, United States

For generation IV reactors, a Ferritic/Martensitic steel known as HT9 is a strong candidate for cladding and structural component materials [1]. Being so close to the nuclear core, cladding and other structural materials will need to be able to withstand the harsh temperature and radiation field. Specifically, the mechanical properties of a candidate material need to be tested at various doses of irradiation to ensure the safety of the reactor and containment of radioactive fuel. Irradiation through neutron damage can often be time consuming and can result in radioactive material through activation [2]. Ion irradiation can be used to overcome the problems associated with neutron irradiation and can easily be controlled in terms of energy and flux via magnetic fields [2]; however, ion irradiation falls short in terms of penetration depth which is limited [2]. The limited depth of ions for irradiation makes bulk mechanical testing impracticable. Hardness test can be used to obtain some mechanical properties of irradiated materials, but does not have the ability to view the response of the irradiated microstructure under mechanical load [3]. In situ micropillar compression tests can be utilized to study the evolution of the microstructure during mechanical testing. The purpose of the study was to conduct micropillar compression tests on ion irradiated HT9 under Transmission Electron Microscopy (TEM) observation.

\section{Micropillar Fabrication and Testing}

Micropillars were fabricated using Focused Ion Beam (FIB) techniques which were used to both shape the pillars as well as make them electron transparent for TEM observations. Pillars were shaped to be around $300 \mathrm{~nm}$ wide, $900 \mathrm{~nm}$ tall, and $100 \mathrm{~nm}$ thick. Energy Filter Transmission Electron Microscopy (EFTEM) was utilized to ascertain the thicknesses of each pillar which was averaged to determine the stress on the material. Ion Irradiation was performed at Sandia National Laboratories Ion Beam Laboratory. Pillars were subjected to a $1.7 \mathrm{MeV} \mathrm{Au}{ }^{3+}$ ion at a flux of approximately $8.9 \times 10^{11}$ ions $/ \mathrm{cm}^{2}$. Pillar compression tests were conducted using a PI-95 Picoindenter. The PI-95 offers in situ mechanical testing, while measuring the load under constant displacement. Heating during irradiation was performed using a double tilt Gatan $(\bigodot$ heating holder or with a Microelectromechanical System (MEMS) heater that attaches onto the PI-95 holder (limited to $300{ }^{\circ} \mathrm{C}$ ). The effects of dose, irradiation temperature, compression temperature, and strain rate were investigated. Furthermore, $10 \mathrm{keV} \mathrm{He}^{+}$was implanted simultaneously during ion irradiation using a defocused helium beam to observe the effects of helium bubbles on the mechanical properties of the pillars.

\section{Irradiation Induced Defects and Hardening}

Under TEM observations, irradiation induced defects formed and disappeared. Irradiation defects size was seen to have increased with increasing dose as shown in Figure 1. Furthermore, the pillars implanted with $2000 \mathrm{appm} / \mathrm{dpa}$ of helium showed the presence of cavities. Mechanical testing using the PI-95 was conducted once a certain dose was reached. The load as a function of displacement curves were recorded during the experiment and were converted into stress strain curves. Videos of the compression test were recorded to observe the behavior of the microstructure under compression. An example of one of the stress strain curves after reaching an irradiation dose of $4 \mathrm{dpa}$ at with micrographs at various strain values is shown in Figure 2. The stress strain curves show indications of load drops. Besides the acute load drops 
in the curves, many of the curves have a steady drop in stress over a range of strains. The steady load drops are from the bending of the pillars. Due to their thin geometry to remain electron transparent, some pillars tended to bend instead of compress over time. One observation that can be made from the stress strain curves is the increase in the mechanical strength as a function of dose that is obtained during the pillar compression test. The increase in the strength of the material from irradiation, known as irradiation hardening, is a common phenomenon that happens to irradiated metals $[4,5]$. Irradiation induced defects provide obstacles to the microstructure that hinder dislocation motion [6].

$\mathrm{O}$ dpa \& $\mathrm{O}$ appm He

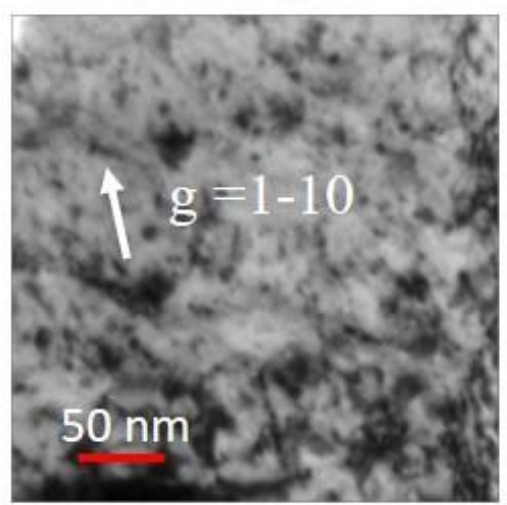

10 dpa \& 1000 appm He

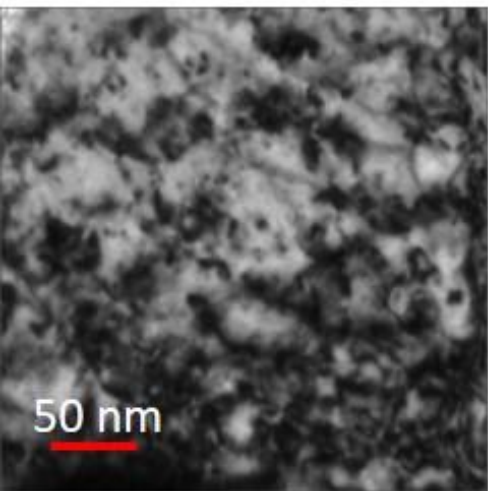

2 dpa \& 200 appm He

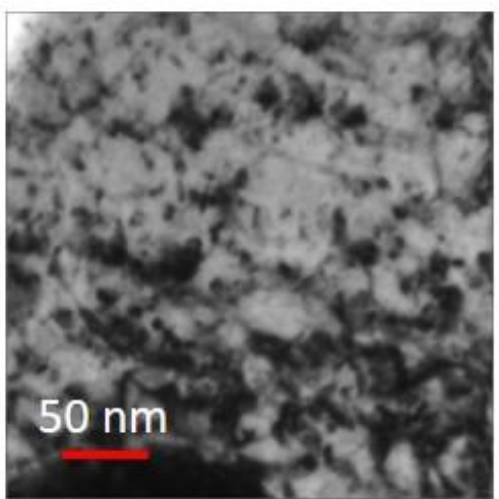

15 dpa \& 1500 appm He

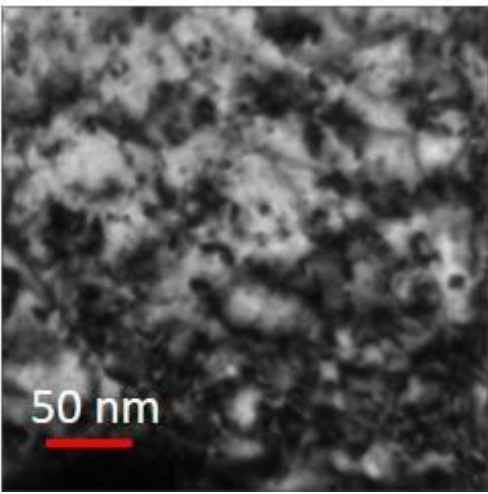

4 dpa \& 400 appm He

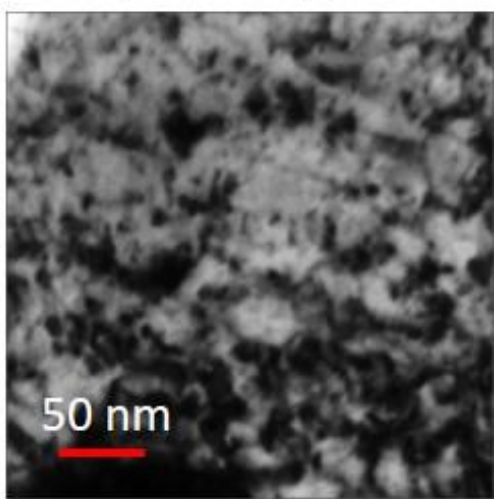

20 dpa \& 2000 appm He

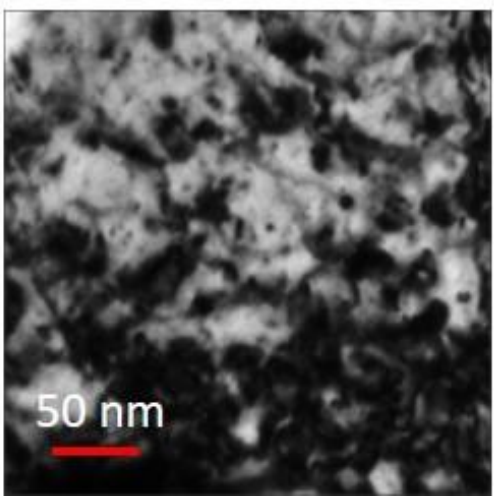

Figure 1. Evolution of HT9 Microstructure under Gold Ion Irradiation and Helium Implantation
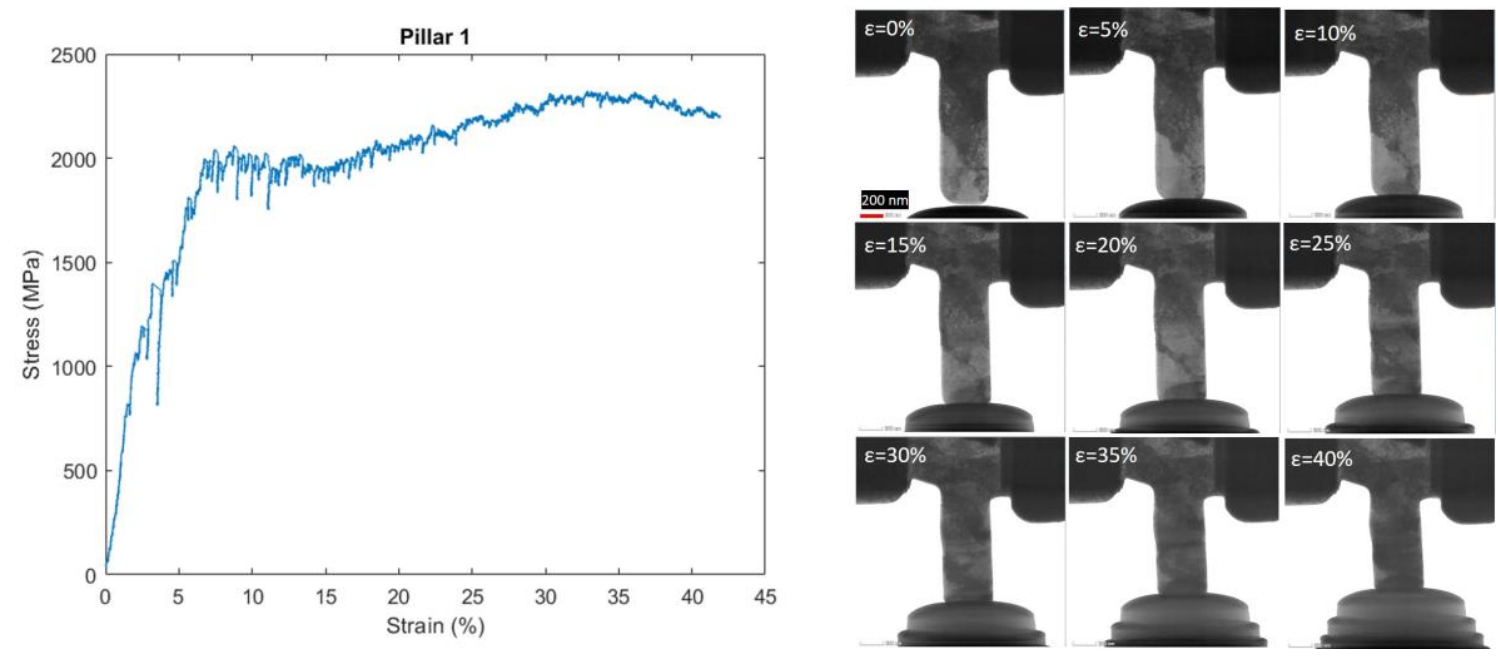
Figure 2. Stress Strain Curves of the HT9 Pillars after 4 dpa and Micrographs of Pillars at various Strains

References

1. Klueh, R.L. and A.T. Nelson, Ferritic/martensitic steels for next-generation reactors. Journal of Nuclear Materials, 2007. 371(1): p. 37-52.

2. Ribis, J., et al., Comparison of the neutron and ion irradiation response of nano-oxides in oxide dispersion strengthened materials. Journal of Materials Research, 2015. 30(14): p. 2210-2221.

3. Heintze, C., et al., Irradiation hardening of Fe-9Cr-based alloys and ODS Eurofer: Effect of helium implantation and iron-ion irradiation at $300{ }^{\circ} \mathrm{C}$ including sequence effects. Journal of Nuclear Materials, 2016. 470: p. 258-267.

4. Gao, J., K. Yabuuchi, and A. Kimura, Ion-irradiation hardening and microstructural evolution in F82H and ferritic alloys. Journal of Nuclear Materials, 2019. 515: p. 294-302.

5. Heintze, C., et al., Ion irradiation combined with nanoindentation as a screening test procedure for irradiation hardening. Journal of Nuclear Materials, 2016. 472: p. 196-205.

6. This work was supported by the U.S. Department of Energy, Office of Nuclear Energy under DOE Idaho Operations Office Contract DE-AC07- 051D14517 as part of a Nuclear Science User Facilities experiment. This work was performed, in part, at the Center for Integrated Nanotechnologies, an Office of Science User Facility operated for the U.S. Department of Energy (DOE) Office of Science. Sandia National Laboratories is a multimission laboratory managed and operated by National Technology \& Engineering Solutions of Sandia, LLC, a wholly owned subsidiary of Honeywell International, Inc., for the U.S. DOE's National Nuclear Security Administration under contract DE-NA-0003525. The views expressed in the article do not necessarily represent the views of the U.S. DOE or the United States Government. 